\title{
SHISA3 is differentially expressed in the lymph nodes of patients with metastatic breast cancer.
}

Shahan Mamoor, MS ${ }^{1}$

$3 \quad$ shahanmamoor@gmail.com

East Islip, NY USA

Metastasis to the brain is a clinical problem in patients with breast cancer ${ }^{1-3}$. Between the breast and the brain reside the secondary lymphoid organ, the lymph nodes. We mined published microarray data ${ }^{4,5}$ to compare primary and metastatic tumor transcriptomes for the discovery of genes associated with metastasis to the lymph nodes in humans with metastatic breast cancer. We found that shisa family member 3, SHISA3, was among the genes whose expression was most different in the lymph node metastases of patients with metastatic breast cancer as compared to primary tumors of the breast. Analysis of a separate microarray dataset revealed that SHISA3 was also differentially expressed in brain metastatic tissues. SHISA3 mRNA was present at increased quantities in lymph node metastases as compared to primary tumors of the breast. Importantly, expression of SHISA3 in primary tumors of the breast was correlated with patient overall survival, more significantly in lymph node negative patients than in lymph node positive patients. Modulation of SHISA3 expression may be relevant to the biology by which tumor cells metastasize from the breast to the lymph nodes and the brain in humans with metastatic breast cancer.

Keywords: breast cancer, metastasis, brain metastasis, central nervous system metastasis, lymph node metastasis, shisa family member 3, SHISA3, systems biology of breast cancer, targeted therapeutics in 
One report described a 34\% incidence of central nervous system metastases in patients treated with trastuzumab for breast cancer ${ }^{2}$. More recently, the NEfERT-T clinical trial ${ }^{6}$ which compared administration of either neratinib or trastuzumab in conjunction with paclitaxel demonstrated that in a randomized, controlled setting, in breast cancer patients treated with neratinib, not only was the incidence of central nervous system recurrence significantly lower, the time to central nervous system metastasis was significantly delayed as compared to patients administered trastuzumab ${ }^{6}$. The alarmingly high rate of central nervous system metastasis described, as well as data, both anecdotal ${ }^{2}$ and from a randomized, controlled setting ${ }^{6}$ illustrating that treatment with trastuzumab may be associated with these events demands an enhanced understanding of the transcriptional makeup of brain and lymph node metastatic tissues to support identification of therapeutic targets, whether they are treatment related or not. The lymph nodes reside between the breast and the brain. We performed a global comparative analysis of lymph node metastatic tumor tissues in patients with metastatic breast cancer, compared to primary tumors and normal breast tissues ${ }^{4,5}$. We discovered transcriptome-wide differential expression of the gene encoding shisa family member 3 , SHISA3, in lymph node metastatic tissues of patients with metastatic breast cancer.

\section{Methods}

We used datasets GSE10893 ${ }^{4}$ and GSE52604 5 for this global differential gene expression analysis of brain metastatic breast cancer in conjunction with GEO2R. GSE10893 was generated using Agilent-011521 Human 1A Microarray G4110A technology with $n=71$ primary breast tumors and $n=7$ lymph node metastases from patients with breast cancer; analysis was performed using platform GPL887. GSE52604 was generated using Agilent-014850 Whole Human Genome Microarray 4x44K G4112F with $n=10$ normal breast tissues and $n=35$ brain metastases from patients with breast cancer; analysis was performed using platform GPL6480. The Benjamini and Hochberg method of $p$-value adjustment was used for ranking of differential expression but raw $p$-values were used to assess statistical significance of global differential expression. Log-transformation of data was auto-detected, and the NCBI generated category of platform annotation was used. A statistical test was performed to evaluate whether SHISA3 gene expression was significantly different between primary tumors of the breast and lymph node metastases in humans with breast cancer using a two-tailed t-test. For Kaplan-Meier survival analysis, we used the Kaplan-Meier plotter online tool ${ }^{7}$ for correlation of SHISA3 mRNA expression levels with overall survival in $n=1402$ breast cancer patients.

\section{$\underline{\text { Results }}$}

We performed global comparative transcriptome analysis of metastatic tumor tissues of patients with metastatic breast cancer using published microarray data ${ }^{4,5}$ to describe the transcriptional landscape of metastasis in human breast cancer in an unbiased fashion and for the discovery of novel therapeutic targets.

\section{SHISA3 is differentially expressed in the lymph node metastases of patients with metastatic breast cancer.}

Through blind, systems-level analysis of published microarray data ${ }^{4}$, we identified shisa family member 3, encoded by SHISA3, as a differentially expressed gene in the lymph node metastatic tissues of humans with breast cancer (Table 1). When sorting each of the genes expressed in lymph node metastases based on significance of difference as compared to primary tumors of the breast in patients with breast cancer, SHISA3 ranked 11 out of 18034 total transcripts (Chart 1), equating to 99.9\% differential expression. Differential expression of SHISA3 in the lymph node metastases of patients with metastatic breast cancer was statistically significant (Chart $1 ; p=1.13 \mathrm{e}-05$ ). 
Differential gene expression analyses that utilize primary and metastatic tumor tissues to discover genes associated with metastasis can be challenging due to the relatively heterogenous cellular composition and constitution of the tumor as compared to normal, untransformed (benign) tissues. Thus, to attempt to validate differential transcriptome-wide differential expression of SHISA3 in metastatic tissues in human breast cancer, we queried a second microarray dataset ${ }^{5}$, here comparing normal breast tissues rather than primary tumors of the breast, to brain metastases. Again, we identified SHISA3 as a differentially expressed gene in the metastatic tissues of humans with breast cancer, here, in the brain metastatic tissues of patients with breast cancer (Chart 2). When sorting each of the genes expressed in brain metastases based on significance of difference as compared to normal breast tissues, SHISA3 ranked 13313 out of 41093 total transcripts (Chart 2), equating to $67.6 \%$ differential expression. Differential expression of SHISA3 in the brain metastases of patients with metastatic breast cancer was statistically significant (Chart 2; $p=1.72 \mathrm{e}-02$ ). Thus, differential expression of SHISA3, transcriptome-wide, in the metastatic tissues of women with metastatic breast cancer was conserved across two independent microarray datasets, both in lymph node and brain metastases. This suggests that SHISA3 differential expression in human metastatic breast cancer is a bona fide biological process and that differential expression of SHISA3 in metastatic breast cancer is shared between the brain and the lymph nodes.

SHISA3 is expressed at higher levels in the lymph node metastases of patients with metastatic breast cancer.

We obtained exact mRNA expression levels for SHISA3, in primary tumors of the breast and in lymph node metastases of patients with metastatic breast cancer to determine direction and statistical significance of change in SHISA3 expression in lymph node metastatic tissues. We observed significantly increased expression of SHISA3 in the lymph node metastases of patients with breast cancer as compared to primary tumors of the breast: SHISA3 was expressed at $-1.31 \pm 0.88$ arbitrary units (AU) in primary tumors of the breast, while it was expressed at $0.37 \pm 1.24 \mathrm{AU}$ in lymph node metastatic tissues (Figure 1). The difference in SHISA3 mRNA levels between primary tumors of the breast and lymph node metastases was statistically significant (Figure $1 ; p=0.000015$ ).

\section{SHISA3 expression is significantly correlated with survival outcomes in human breast cancer.}

We performed Kaplan-Meier survival analysis ${ }^{7}$ in $n=1402$ breast cancer patients in total, to evaluate whether SHISA3 tumor expression was correlated with survival outcomes in breast cancer. We observed a statistically significant correlation between primary tumor expression of SHISA3 and overall survival (OS) in patients with breast cancer (Figure 2). Patients whose primary tumors expressed low levels of SHISA3 possessed median OS of 70.68 months, while patients whose tumors expressed high levels of SHISA3 possessed median OS of 136.8 months. This difference in OS based on SHISA3 tumor expression in patients with breast cancer was statistically significant (Figure 2, Chart 3; logrank $p$-value: 0.018; hazard ratio: $0.68(0.5-0.94))$.

We also performed Kaplan-Meier survival analysis ${ }^{7}$ in $n=313$ lymph node positive breast cancer patients and $n=594$ lymph node negative breast cancer patients, to evaluate whether lymph node status influenced correlation of SHISA3 tumor expression with survival outcomes in human breast cancer. We observed a correlation between primary tumor expression of SHISA3 and overall survival (OS) in lymph node positive breast cancer (Figure 2). Lymph node positive patients whose primary tumors expressed low levels of SHISA3 possessed median OS of 63.36 months, while lymph node positive patients whose tumors expressed high levels of SHISA3 possessed median OS of 40.53 months. This difference in OS based on SHISA3 tumor expression in lymph node positive patients with breast cancer trended towards statistical significance (Figure 2, Chart 3; logrank $p$-value: 0.1; hazard ratio: 1.55 (0.91-2.65)). Thus, overall survival based on SHISA3 primary tumor expression was markedly shorter in lymph node positive patients than in the general breast cancer patient population. 
SHISA3 primary tumor expression was not significantly correlated with overall survival outcomes in lymph node negative patients (Figure 2, Chart 3; logrank $p$-value: 0.94; hazard ratio: 0.96(0.39-2.37)).

Thus, by mining published microarray data ${ }^{4,5}$ in an unbiased and systematic fashion, we identified SHISA3 as among the genes whose expression was most different, transcriptome-wide, in the lymph node metastases of patients with breast cancer, when compared to primary tumors; we observed significantly increased expression of SHISA3 in lymph node metastases as compared to primary tumors of the breast. Further, we found a significant correlation between SHISA3 expression and patient survival outcomes, as

\section{$\underline{\text { Discussion }}$}

We provided evidence here that shisa family member 3, SHISA3, is among the genes whose expression is most different in the lymph node metastases of patients with metastatic breast cancer as compared to primary tumors of the breast, that SHISA3 is also differentially expressed in brain metastatic tissues in human breast cancer, that SHISA3 mRNA is present at significantly increased quantities in lymph node metastatic tissues as compared to primary tumors of the breast, and that primary tumor SHISA3 expression is significantly correlated with patient survival outcomes in human breast cancer, markedly more so in lymph node positive patients than in lymph node negative patients. Evaluation of the effects of genetic depletion of SHISA3 in mouse models of metastatic breast cancer on metastasis to the central nervous system is merited. Modulation of SHISA3 expression may be relevant to the processes by which breast cancer cells exit the breast, enter the vasculature and/or lymphatics, reside in the lymph nodes, evade immune clearance, breach the blood-brain barrier and colonize the brain. 


\section{$\underline{\text { References }}$}

1. Lin, N.U., Amiri-Kordestani, L., Palmieri, D., Liewehr, D.J. and Steeg, P.S., 2013. CNS metastases in breast cancer: old challenge, new frontiers.

2. Bendell, J.C., Domchek, S.M., Burstein, H.J., Harris, L., Younger, J., Kuter, I., Bunnell, C., Rue, M., Gelman, R. and Winer, E., 2003. Central nervous system metastases in women who receive trastuzumab-based therapy for metastatic breast carcinoma. Cancer, 97(12), pp.2972-2977.

3. Tsukada, Y., Fouad, A., Pickren, J.W. and Lane, W.W., 1983. Central nervous system metastasis from breast carcinoma autopsy study. Cancer, 52(12), pp.2349-2354.

4. Weigman, V.J., Chao, H.H., Shabalin, A.A., He, X., Parker, J.S., Nordgard, S.H., Grushko, T., Huo, D., Nwachukwu, C., Nobel, A. and Kristensen, V.N., 2012. Basal-like Breast cancer DNA copy number losses identify genes involved in genomic instability, response to therapy, and patient survival. Breast cancer research and treatment, 133(3), pp.865-880.

5. Salhia, B., Kiefer, J., Ross, J.T., Metapally, R., Martinez, R.A., Johnson, K.N., DiPerna, D.M., Paquette, K.M., Jung, S., Nasser, S. and Wallstrom, G., 2014. Integrated genomic and epigenomic analysis of breast cancer brain metastasis. Plos one, $9(1)$, p.e85448.

6. Awada, A., Colomer, R., Inoue, K., Bondarenko, I., Badwe, R.A., Demetriou, G., Lee, S.C., Mehta, A.O., Kim, S.B., Bachelot, T. and Goswami, C., 2016. Neratinib plus paclitaxel vs trastuzumab plus paclitaxel in previously untreated metastatic ERBB2-positive breast cancer: the NEfERT-T randomized clinical trial. JAMA oncology, 2(12), pp.1557-1564.

7. Györffy, B., Lanczky, A., Eklund, A.C., Denkert, C., Budczies, J., Li, Q. and Szallasi, Z., 2010. An online survival analysis tool to rapidly assess the effect of 22,277 genes on breast cancer prognosis using microarray data of 1,809 patients. Breast cancer research and treatment, 123(3), pp.725-731. 


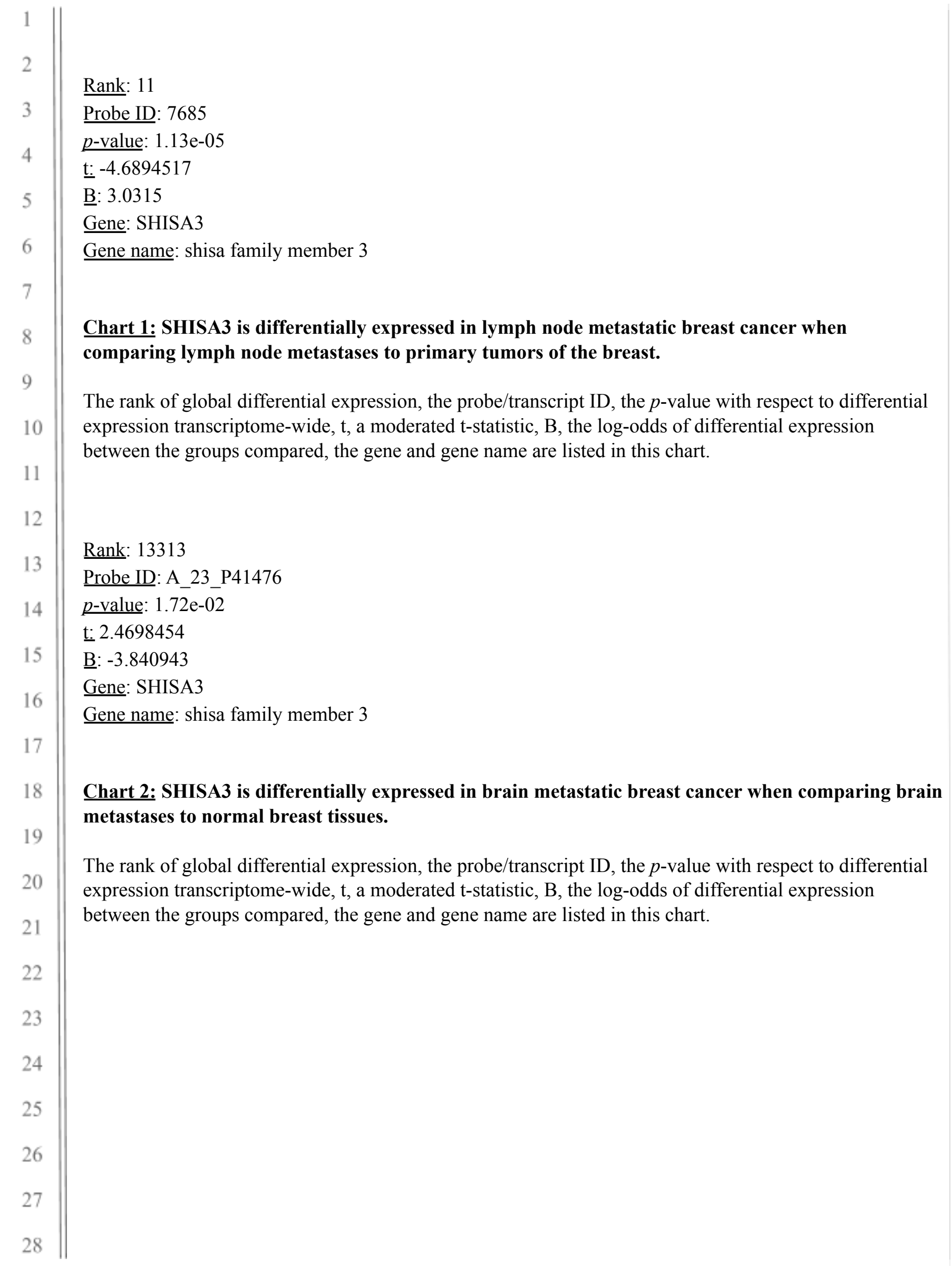




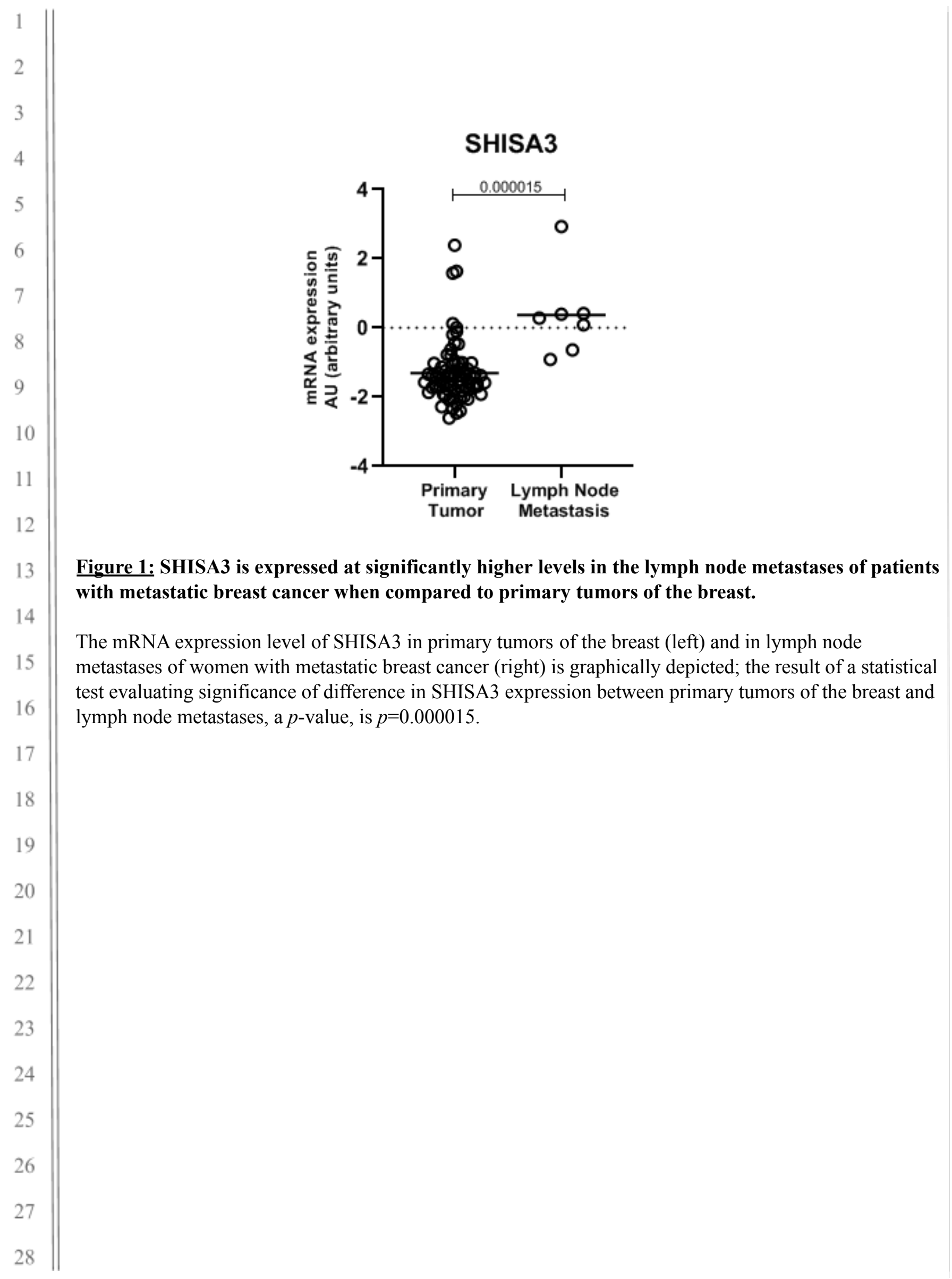




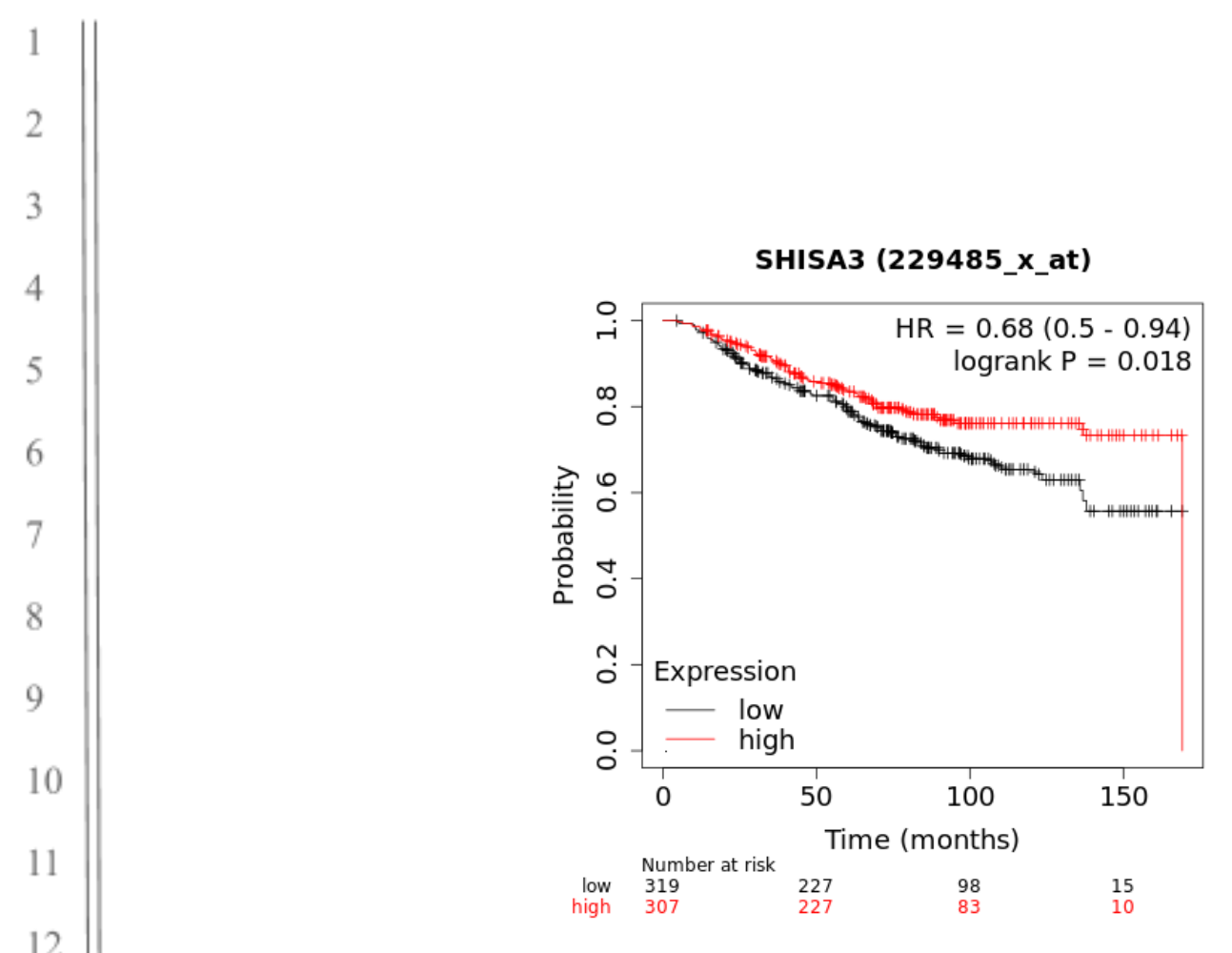

Figure 2: Significant correlation between SHISA3 primary tumor expression and overall survival in patients with breast cancer, which is markedly more significant in lymph node negative patients than in lymph node positive patients.

Depicted in this Kaplan-Meier plot (above) is the probability of overall survival for $n=1402$ total patients stratified into two groups, based on low or high expression of SHISA3 in patient primary tumors. The log rank $p$-value denoting statistical significance of difference in overall survival when comparing the two groups, as well as hazard ratio for this comparison is listed above. Listed below is the number of patients at risk (number of patients alive) per interval, after stratification based on SHISA3 expression; in the first interval, number at risk is number of patients alive; in each subsequent interval, number at risk is the number at risk less those who have expired or are censored. Below, Kaplan-Meier plots for probability of overall survival in $n=313$ lymph node positive patients (left) and $n=594$ lymph node negative patients (right).

SHISA3 (229485_x_at)

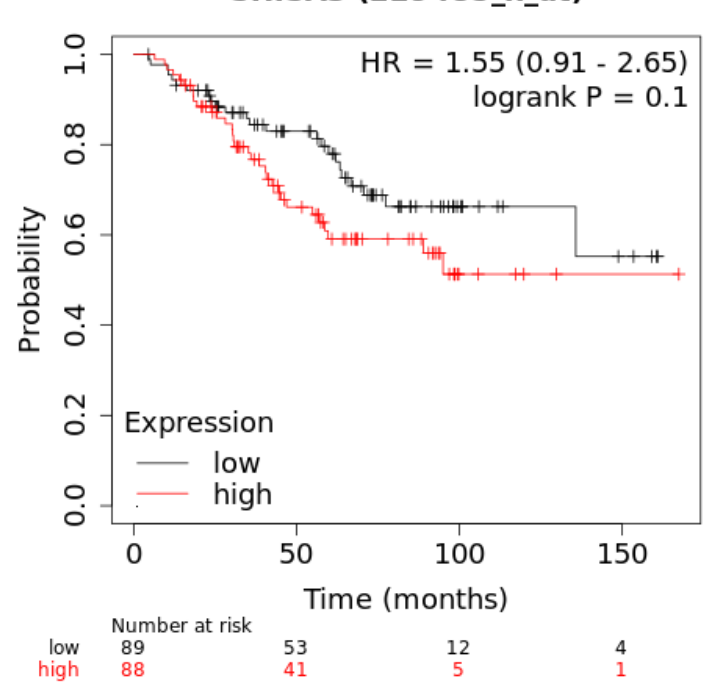

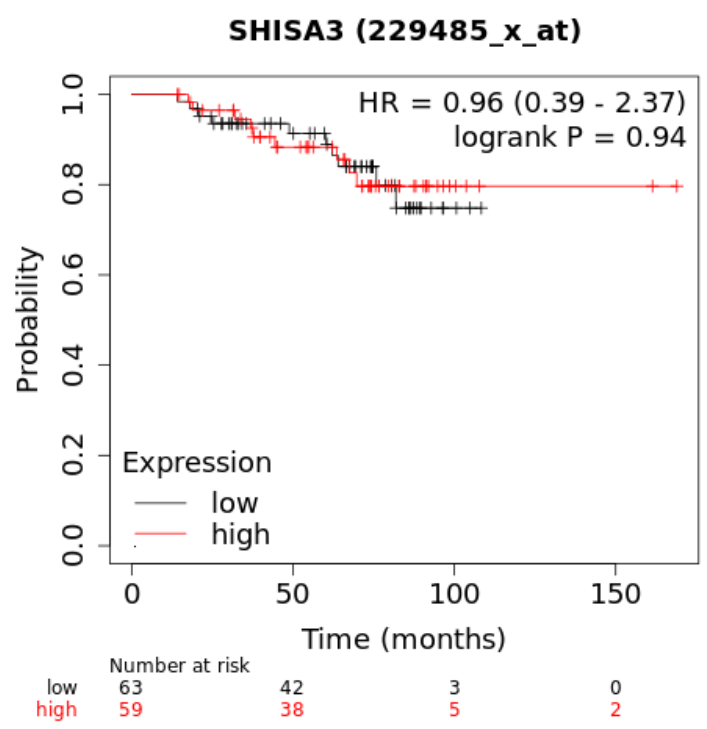




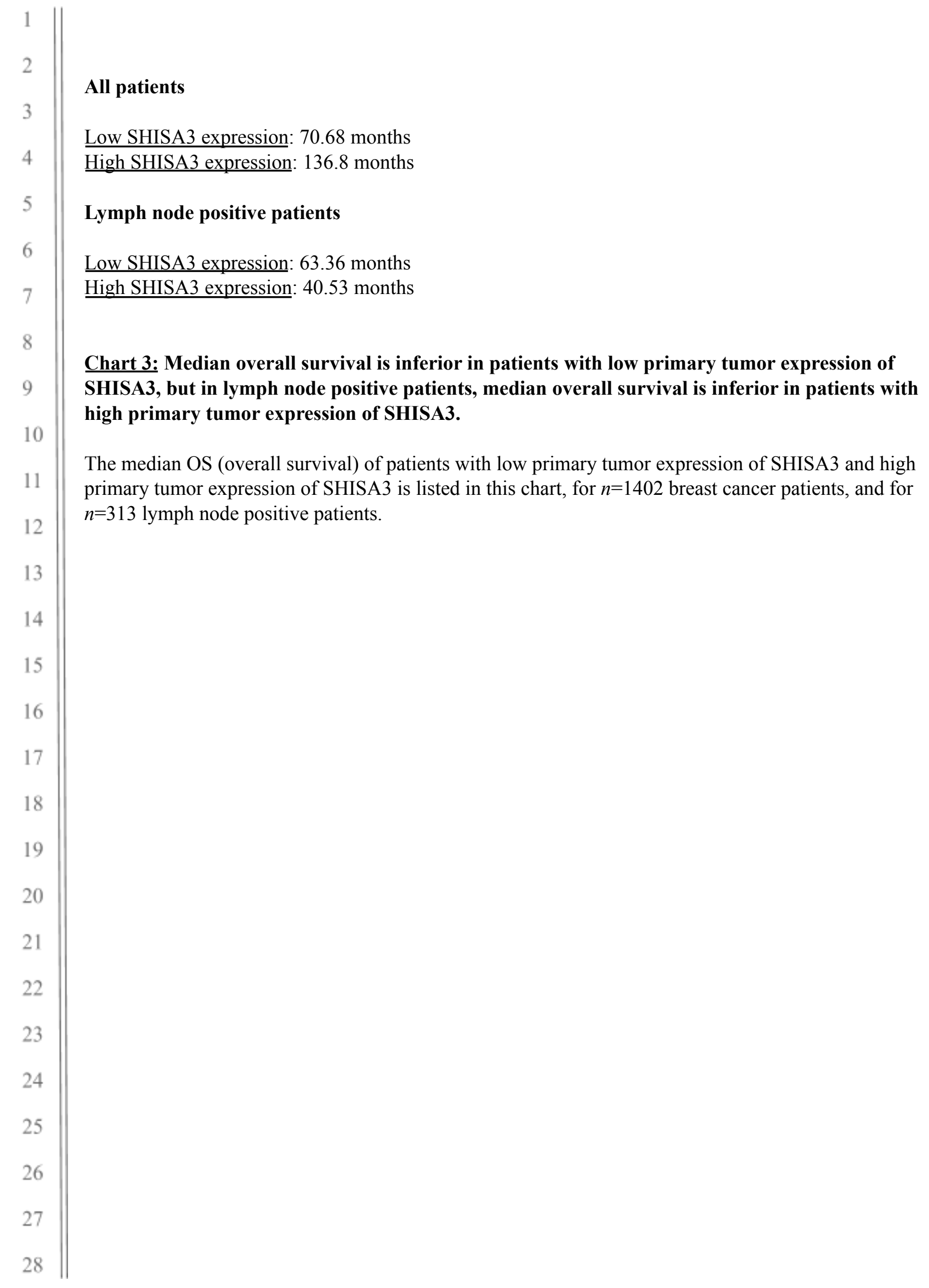

